\title{
System outage probability analysis in uplink multi-hop cellular systems over composite channels
}

\author{
Xibin Zhao ${ }^{1,2,3}$, Jun-Bo Wang ${ }^{1,4,5^{*}}$, Jin-Yuan Wang ${ }^{4}$, Ming Chen ${ }^{5}$, Min Feng ${ }^{4}$ and Ming Sheng ${ }^{4}$
}

\begin{abstract}
Owing to its superior performances, the multi-hop cellular system has drawn much attention in recent years. This paper aims to study the uplink system outage probability of the multi-hop cellular system over composite channels. Initially, we consider a composite channel model, which takes path loss, lognormal shadowing and Nakagami-m fading into account. Then, based on the amplify-and-forward relaying, the signal-to-noise ratio of each link is investigated. Further, an expression of the outage probability for a mobile station (MS) over a given position is derived after employing selective transmission scheme. After that, considering the distribution of MSs in the cellular systems, a numerical expression of the system outage probability is further obtained. Numerical results prove that the derived expression of the system outage probability can provide very good approximation to the realistic outage performance without time-intensive simulations. Moreover, it's also shown that the muilti-hop cellular system in this paper outperforms the conventional cellular system in terms of outage probability.
\end{abstract}

Keywords: multi-hop cellular system, system outage probability, composite channel, amplify-and-forward relaying, selective transmission

\section{Introduction}

The next generation wireless communication systems will provide very high data rates and support various multimedia applications. However, due to the limitation of the available transmission resources, the inherent problems of limited capacity and coverage in conventional cellular system are hard to overcome. In the past few years, there has been increasing interest in the study of the multi-hop cellular system [1]. Unlike the conventional cellular system, data packets in multi-hop cellular system, in addition to being transmitted directly between a mobile station (MS) and the base station (BS), can also be indirectly transmitted hop by hop with the help of relay stations (RSs). Recent studies have shown that this feature of the multi-hop cellular system can enhance coverage [2], improve system throughput [3], reduce transmission power $[4,5]$, etc. Without any doubt, the multi-hop cellular system will become a very

\footnotetext{
* Correspondence: jbwang@nuaa.edu.cn

${ }^{1}$ Key Laboratory for Information System Security of Ministry of Education,

School of Software, Tsinghua University, Beijing 100084, China

Full list of author information is available at the end of the article
}

promising candidate in future wireless communication system.

System outage probability is an important indicator in wireless communication systems, and relay-assisted transmission has the advantage of extending coverage without high power usage at the transmitter. Up to now, some works have been done to analyze the outage performance in relay-assisted communication system. The authors in [6-9] analyzed the outage performance in two-hop relay-assisted systems with one RS. In [10], the outage probability was further studied in a two-hop system with one RS and with multiple antennas at the transmitter. In [11-15], two-hop relay-assisted systems with multiple RSs were discussed. Then, multi-hop relay-assisted systems with multiple RSs were investigated in [16-20]. In [21], a thorough discussion of the two-hop system was presented, where many of the previous results for this system were summarized. Also, a brief discussion of the multi-hop system was included. Recently, in [22], the work in [21] was generalized by analyzing the outage probability of the multi-hop system. It should be noted that, most of these previous

\section{SpringerOpen ${ }^{\circ}$}

(C) 2011 Zhao et al; licensee Springer. This is an Open Access article distributed under the terms of the Creative Commons Attribution License (http://creativecommons.org/licenses/by/2.0), which permits unrestricted use, distribution, and reproduction in any medium, provided the original work is properly cited. 
works were based on Rayleigh fading channels [7-9,11-15,18-20,22]. However, Rayleigh fading is just a special case of Nakagami- $m$ fading. As is well known, Nakagami- $m$ fading represents a wide range of realistic fading conditions and fits experimental data. Therefore, the Rayleigh fading channel lacks generality. In addition, all of these previous works, [6-22], did not focus on the multi-hop cellular system scenario, and therefore these literatures did not consider the impact of the distribution of MS on system performance. However, there has been insufficient works done on the outage performance analysis in multi-hop cellular system. The authors in [23] analyzed the outage probability and spectral efficiency in multi-hop cellular system with uniform MS distribution. However, the uniform MS distribution may not be a practical situation for dense urban scenario in multi-hop cellular system, as the MSs may be clustered into hot zone. Thus, the obtained results cannot be applied to evaluate the outage performance under various scenarios. To the best of the authors' knowledge, the system outage probability problems in multi-hop cellular system have not been completely discussed in open literatures, so it is interesting and necessary to study these problems.

In this paper, we are motivated to study the system outage probability in multi-hop cellular system over composite channels. Initially, considering the characteristic of practical wireless propagation environments, a more general channel model is established, which takes path loss, lognormal shadowing and Nakagami- $m$ fading into consideration. Then, by employing the selective transmission (ST) scheme, the best channel will be selected from multiple available channels for transmission by the criterion of maximizing the output signal-tonoise ratio (SNR) at the receiver. Moreover, to reduce the computational complexity, a numerical expression of the outage probability is derived, and it's very easy to evaluate the outage performance. Furthermore, in order to investigate the impact of the distributions of MS in the cellular system on system performance, we introduce a probability density function (PDF) of the distribution of MS into the final theoretical expression of the system outage probability. The PDF proposed in this paper is more general to describe the distribution of MS, which is suitable for MS uniform distribution as well as non-uniform distribution.

The remainder of this paper is organized as follows. The system model of the uplink multi-hop cellular system is described in the next section. In Section 3, a numerical expression of the system outage probability is derived after employing ST scheme at the transmitter. Numerical results are presented in Section 4 before conclusions are drawn in Section 5.

\section{System model}

Consider a single cell multi-hop cellular system, as shown in Figure 1. Assume that the radius of the cell is $R$. The BS is in the center of the cell. The $N$ relay stations are placed arbitrarily in the cell, which can be denoted as RSi $(i=1,2, \ldots, N)$. Due to the implementation limitation, only one antenna is available at the MS and each RS. Without loss of generality, the positions of the MS, BS and RSi are denoted by the polar coordinates $(\rho, \theta),(0,0)$ and $\left(A_{i}, \alpha_{i}\right)$, respectively. Note that $\rho$ and $\theta$ are the distance and angle of the MS relative to the cell center, while $A_{i}$ and $\alpha_{i}$ are the distance and angle of RSi to the cell center.

Here, we only consider the uplink transmission. In this system, the MS can transmit information to the BS directly or indirectly with the help of the RS by using two hops. Therefore, there are total $N+1$ channels, i.e., one direct transmission channel and $N$ relay transmission channels, can be used to transmit information. Suppose that the channel state information (CSI) is known at the transmitter side, and the ST scheme can be applied to select one channel from the $N+1$ channels for transmission by the criterion of maximizing the output SNR at the receiver.

It should be noted that the direct transmission channel is also named as the MS-to-BS link, while each relay transmission channel contains one MS-to-RS link and one RS-to-BS link. Therefore, there are total $2 N+1$ links in this system, i.e., one MS-to-BS link, $N$ MS-toRS links, and $N$ RS-to-BS links. Assume all the link gains undergo mutually independent non-identical

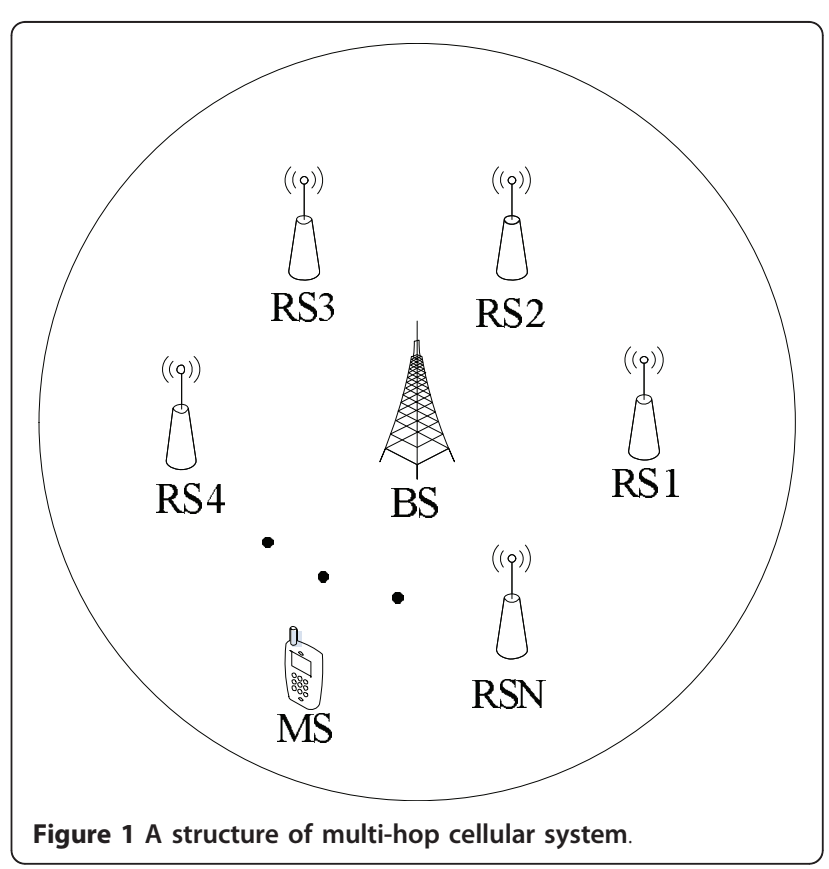


shadowed Nakagami distributions. Therefore, the link gains can be denoted as

$$
h_{i}=\sqrt{\Omega_{i}} g_{i}, \quad \forall i \in\{0,1,2, \ldots, 2 N\}
$$

where $h_{0}, h_{i}(i=1,2, \ldots, N)$, and $h_{i}(i=N+1, N+2, \ldots$, $2 N)$ denote the link gains for the MS-to-BS link, the MS-to-RS links, and the RS-to-BS links, respectively.

In (1), $\Omega_{i}$ represents the lognormal shadowing, which can be modeled by a lognormal distribution [24]

$$
f\left(\Omega_{i}\right)=\frac{\xi}{\sqrt{2 \pi} \sigma_{i} \Omega_{i}} \exp \left\{-\frac{\left[10 \log _{10} \Omega_{i}-\omega_{i}(\rho, \theta)\right]^{2}}{2 \sigma_{i}^{2}}\right\}
$$

where $\xi=10 / \ln 10 . \omega_{i}(\rho, \theta)$ (in $\mathrm{dB}$ ) and $\sigma_{i}$ (in $\mathrm{dB}$ ) are the mean and standard derivation of $10 \log _{10} \Omega_{i}$, respectively. It should be noted that $\omega_{i}(\rho, \theta)$ is a function of MS's position, and it is determined by the path loss of each link

$$
\omega_{i}(\rho, \theta)=10 \log _{10}\left[\frac{d_{0}}{d_{i}(\rho, \theta)}\right]^{\beta_{i}}
$$

where $d_{0}$ is the reference distance, and $\beta_{i}$ represents the path loss exponent. $d_{i}(\rho, \theta)$ denotes the length of each link, which can be formulated as

$$
d_{i}(\rho, \theta)= \begin{cases}\rho & \text { for } i=0 \\ \sqrt{\rho^{2}+A_{i}^{2}-2 \rho A_{i} \cos \left(\theta-\alpha_{i}\right)} & \text { for } i=1,2, \ldots, N \\ A_{i} & \text { for } i=N+1, \ldots, 2 N\end{cases}
$$

Further, $g_{i}$ in (1) denotes the fast fading and its envelope follows an independent but not identical Nakagami distribution, so the PDF of $\left|g_{i}\right|$ can be written as [25]

$$
f\left(\left|g_{i}\right|\right)=\frac{2 m_{i}^{m_{i}}\left|g_{i}\right|^{2 m_{i}-1}}{\Gamma\left(m_{i}\right)} \exp \left(-m_{i}\left|g_{i}\right|^{2}\right)
$$

where $m_{i}$ is the Nakagami parameter and $\Gamma($.$) is the$ gamma function.

\section{System outage probability analysis}

In this section, we perform the outage probability analysis of the uplink multi-hop cellular system. Initially, we derive the output SNR of each link, and then the outage probabilities of direct transmission channel and relay transmission channels are analyzed in turn. Finally, by using the ST scheme and considering the distribution of MSs in the cell, the system outage probability is derived.

\subsection{Output SNR}

Assume that the transmit power of the MS is $E_{\mathrm{M}}$. For the relay transmission channels, amplify-and-forward (AF) relaying is employed, that is, after receiving the signal from MS, each RS will retransmit the received signal to the $\mathrm{BS}$ with the transmit power $E_{\mathrm{R}}$. Therefore, by using (1), the received SNR of each link can be expressed as

$$
\gamma_{i}=\left\{\begin{array}{l}
\frac{E_{\mathrm{M}}\left|h_{i}\right|^{2}}{N_{0}}=\frac{E_{\mathrm{M}} \Omega_{i}\left|g_{i}\right|^{2}}{N_{0}}, i=0,1, \ldots, N \\
\frac{E_{\mathrm{R}}\left|h_{i}\right|^{2}}{N_{0}}=\frac{E_{\mathrm{R}} \Omega_{i}\left|g_{i}\right|^{2}}{N_{0}}, i=N+1, N+2, \ldots, 2 N
\end{array}\right.
$$

Where $N_{0}$ is the background noise power. $\gamma_{0}$ denotes the SNR of the MS-to-BS link, $\gamma_{1}, \ldots, \gamma_{N}$ are the SNR of the MS-to-RS links, and $\gamma_{N+1}, \ldots, \gamma_{2 N}$ represent the SNR of the RS-to-BS links.

\subsection{Outage probability of direct transmission channel}

Since the envelope of $g_{i}$ undergoes Nakagami- $m$ distribution, it can be known from [25] that the square envelope $\left|g_{i}\right|^{2}$ follow gamma distribution. Therefore, $\gamma_{i}$ in (6) are both Gamma-lognormal distributed and their PDFs can be given by

$$
f\left(\gamma_{i}\right)=\int_{0}^{\infty} \frac{m_{i}^{m} i_{i}^{m_{i}-1}}{S_{i}^{m_{i}} \Gamma\left(m_{i}\right)} \exp \left(-\frac{m_{i} \gamma_{i}}{S_{i}}\right) \frac{\xi}{\sqrt{2 \pi} \sigma_{i} S_{i}} \exp \left[-\frac{\left(10 \log _{10} S_{i}-\mu_{i}(\rho, \theta)\right)^{2}}{2 \sigma_{i}^{2}}\right] \mathrm{d} S_{i}
$$

where

$$
S_{i}=\left\{\begin{array}{l}
\frac{E_{\mathrm{M}} \Omega_{i}}{N_{0}}, \text { for } i=0,1, \ldots, N \\
\frac{E_{\mathrm{R}} \Omega_{i}}{N_{0}}, \text { for } i=N+1, N+2, \ldots, 2 N
\end{array}\right.
$$

and

$$
\mu_{i}(\rho, \theta)=\left\{\begin{array}{l}
\omega_{i}(\rho, \theta)+10 \log _{10}\left(\frac{E_{\mathrm{M}}}{N_{0}}\right), \text { for } i=0,1, \ldots, N \\
\omega_{i}(\rho, \theta)+10 \log _{10}\left(\frac{E_{\mathrm{R}}}{N_{0}}\right), \text { for } i=N+1, N+2, \ldots, 2 N
\end{array}\right.
$$

Assume that $\gamma_{\text {th }}$ is the minimum SNR threshold that guarantees the reliable reception. Therefore, the probability $\operatorname{Pr}\left(\gamma_{i}<\gamma_{\text {th }}\right)$ can be expressed as

$$
\begin{aligned}
\operatorname{Pr}\left(\gamma_{i}<\gamma_{\mathrm{th}}\right)= & \int_{0}^{\gamma_{\mathrm{ht}}} f\left(\gamma_{i}\right) \mathrm{d} \gamma_{i} \\
= & \int_{0}^{\gamma_{\mathrm{h}}} \int_{0}^{\infty} \frac{m_{i}^{m_{i}} \gamma_{i}^{m_{i}-1}}{S_{i}^{m_{i}} \Gamma\left(m_{i}\right)} \exp \left(-\frac{m_{i} \gamma_{i}}{S_{i}}\right) \\
& \times \frac{\xi}{\sqrt{2 \pi} \sigma_{i} S_{i}} \exp \left[-\frac{\left(10 \log _{10} S_{i}-\mu_{i}(\rho, \theta)\right)^{2}}{2 \sigma_{i}^{2}}\right] \mathrm{d} S_{i} \mathrm{~d} \gamma_{i}
\end{aligned}
$$

Then, exchange the integral order and let $x=m_{i} \gamma_{i} / S_{i}$, we can further obtain $\operatorname{Pr}\left(\gamma_{i}<\gamma_{\text {th }}\right)$ as

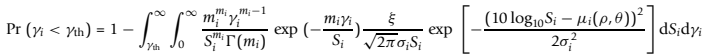

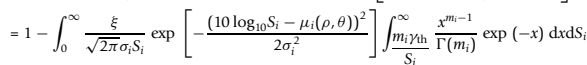

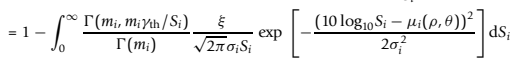

where $\Gamma(n, x)=\int_{x}^{\infty} e^{-t} t^{n-1} \mathrm{~d} t$ is the incomplete gamma function.

Let $x=\left(10 \log _{10} S_{i}-\mu_{i}(\rho, \theta)\right) /\left(\sqrt{2} \sigma_{i}\right)$, and then by using the Gauss-Hermite integral [26], the probability in 
(11) can be written as a simple form

$$
\operatorname{Pr}\left(\gamma_{i}<\gamma_{\mathrm{th}}\right)=1-\frac{1}{\sqrt{\pi} \Gamma\left(m_{i}\right)} \sum_{n=1}^{N_{p}} H_{x_{n}} \Gamma\left[m_{i}, \frac{m_{i} \gamma_{\mathrm{th}}}{10^{\left(\sqrt{2} \sigma_{i} x_{n}+\mu_{i}(\rho, \theta)\right) / 10}}\right]
$$

where $x_{n}$ and $H_{x_{n}}$ are the base point and weight factor of $N_{p}$-order Hermite polynomial, respectively.

To facilitate description, let $\gamma_{0}^{\mathrm{D}}=\gamma_{0}$ denote the output SNR of direct transmission channel. Therefore, the outage probability of direct transmission channel can be derived when $i=0$ in (12) is satisfied, which can be written as

$$
\operatorname{Pr}\left(\gamma_{0}^{\mathrm{D}}<\gamma_{\mathrm{th}}\right)=1-\frac{1}{\sqrt{\pi} \Gamma\left(m_{0}\right)} \sum_{n=1}^{N_{p}} H_{x_{n}} \Gamma\left[m_{0}, \frac{m_{0} \gamma_{\mathrm{th}}}{a_{n}(\rho, \theta)}\right]
$$

where $a_{n}(\rho, \theta)=10^{\left(\sqrt{2} \sigma_{0} x_{n}+\mu_{0}(\rho, \theta)\right) / 10}$, and $m_{0}$ denotes the Nakagami parameter for the direct transmission channel.

\subsection{Outage probability of relay transmission channel}

Each relay transmission channel contains two links, i.e., the MS-to-RS link and the RS-to-BS link. To simplify description, the output SNR at each RS for the MS-toRS link is denoted as $\gamma_{j}^{\prime}=\gamma_{j}, \forall j \in\{1,2, \ldots, N\}$, and the output SNR at BS for the RS-to-BS link can be similarly denoted as $\gamma_{j}^{\prime \prime}=\gamma_{l+N}, \forall j \in\{1,2, \ldots, N\}$. From (6), the output SNR can be rewritten as

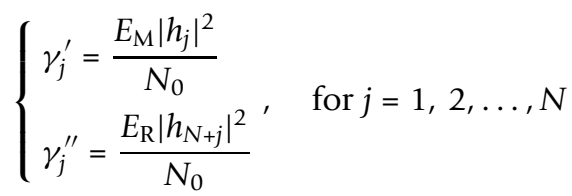

Referring to [27], the equivalent SNR between MS and $\mathrm{BS} \gamma_{j}^{\mathrm{R}}$ can be given by

$$
\gamma_{j}^{\mathrm{R}}=\frac{\gamma_{j}^{\prime} \gamma_{j}^{\prime \prime}}{\gamma_{j}^{\prime}+\gamma_{j}^{\prime \prime}+1}, \text { for } j=1,2, \ldots, N
$$

It can be observed from [28] that $\gamma_{j}^{\mathrm{R}}$ in (15) can be approximated accurately by its upper bound as

$$
\bar{\gamma}_{j}^{\mathrm{R}}=\min \left(\gamma_{j}^{\prime}, \gamma_{j}^{\prime \prime}\right)
$$

Therefore, the outage probability $\operatorname{Pr}\left(\gamma_{j}^{\mathrm{R}}<\gamma_{\text {th }}\right)$ can be expressed as

$$
\begin{aligned}
\operatorname{Pr}\left(\gamma_{j}^{\mathrm{R}}<\gamma_{\mathrm{th}}\right) & \cong \operatorname{Pr}\left(\bar{\gamma}_{j}^{\mathrm{R}}<\gamma_{\mathrm{th}}\right) \\
& =1-\operatorname{Pr}\left(\bar{\gamma}_{j}^{\mathrm{R}} \geq \gamma_{\mathrm{th}}\right) \\
& =1-\operatorname{Pr}\left(\gamma_{j}^{\prime} \geq \gamma_{\mathrm{th}}\right) \operatorname{Pr}\left(\gamma_{j}^{\prime \prime} \geq \gamma_{\mathrm{th}}\right) \\
& =1-\left[1-\operatorname{Pr}\left(\gamma_{j}^{\prime}<\gamma_{\mathrm{th}}\right)\right]\left[1-\operatorname{Pr}\left(\gamma_{j}^{\prime \prime}<\gamma_{\mathrm{th}}\right)\right]
\end{aligned}
$$

Furthermore, from (12), we can easily find that the probability $\operatorname{Pr}\left(\gamma_{j}^{\prime}<\gamma_{\text {th }}\right)$ can be derived as

$$
\operatorname{Pr}\left(\gamma_{j}^{\prime}<\gamma_{\text {th }}\right)=1-\frac{1}{\sqrt{\pi} \Gamma\left(m_{j}^{\prime}\right)} \sum_{n=1}^{N_{p}} H_{x_{n}} \Gamma\left[m_{j^{\prime}} \frac{m_{j}^{\prime} \gamma_{\text {th }}}{b_{n}(\rho, \theta)}\right], \forall j=1,2, \ldots, N
$$

where $b_{n}(\rho, \theta)=10^{\left[\sqrt{2} \sigma_{j} x_{n}+\mu_{j}(\rho, \theta)\right] / 10}$, and $m_{j}^{\prime}=m_{j}$ denotes the Nakagami parameter for the $j^{\text {th }}$ MS-to-RS link.

Owing to the similarity between the MS-to-RS links and the RS-to-BS links, the similar conclusion can be derived from (12) for the RS-to-BS links, so the probability $\operatorname{Pr}\left(\gamma_{j}^{\prime \prime}<\gamma_{\text {th }}\right)$ can be obtained as

$$
\operatorname{Pr}\left(\gamma_{j}^{\prime \prime}<\gamma_{\mathrm{th}}\right)=1-\frac{1}{\sqrt{\pi} \Gamma\left(m_{j}^{\prime \prime}\right)} \sum_{n=1}^{N_{p}} H_{x_{n}} \Gamma\left[m_{j}^{\prime \prime}, \frac{m_{j}^{\prime \prime} \gamma_{\mathrm{h}}}{c_{n}(\rho, \theta)}\right], \forall j=1,2, \ldots, N
$$

Where $c_{n}(\rho, \theta)=10^{\left[\sqrt{2} \sigma_{j+N} x_{n}+\mu j+N(\rho, \theta)\right] / 10}$, and $m_{j}^{\prime \prime}=m_{j+N}$ represents the Nakagami parameter for the $j^{\text {th }}$ RS-to-BS link.

Then, from (17) to (19), the outage probability of the $j^{\text {th }}$ relay transmission channel $\operatorname{Pr}\left(\gamma_{j}^{\mathrm{R}}<\gamma_{\text {th }}\right)$ can be further derived as

$$
\operatorname{Pr}\left(\gamma_{j}^{\mathrm{R}}<\gamma_{\text {th }}\right) \cong 1-\frac{\left\{\sum_{n=1}^{N_{p}} H_{x_{n}} \Gamma\left[m_{j}^{\prime} \frac{m_{j}^{\prime} \gamma_{\text {th }}}{n_{n}(\rho, \theta)}\right]\right\}\left\{\sum_{n=1}^{N_{p}} H_{x_{n}} \Gamma\left[m_{j}^{\prime \prime}, \frac{m_{j}^{\prime \prime} \gamma_{\text {th }}}{c_{n}(\rho, \theta)}\right]\right\}}{\pi \Gamma\left(m_{j}^{\prime}\right) \Gamma\left(m_{j}^{\prime \prime}\right)}
$$

\subsection{System outage probability}

Assume that the CSI is known at the transmitter side, and the ST scheme can be applied to select one channel from the $N+1$ channels for transmission by the criterion of maximizing the output SNR at the receiver. Then, the output SNR $\gamma$ an be given by

$$
\gamma=\max \left\{\gamma_{0}^{\mathrm{D}}, \gamma_{1}^{\mathrm{R}}, \gamma_{2}^{\mathrm{R}}, \ldots, \gamma_{N}^{\mathrm{R}}\right\}
$$

Since all the link gains undergo independent shadowed Nakagami distributions, the output SNRs $\gamma_{0}^{\mathrm{D}}, \gamma_{1}^{\mathrm{R}}, \gamma_{2}^{\mathrm{R}}, \ldots, \gamma_{N}^{\mathrm{R}}$ are independent of each other. Therefore, the outage probability for the MS over a given position can be expressed as

$$
\begin{aligned}
\delta(\rho, \theta) & =\operatorname{Pr}\left(\gamma<\gamma_{\mathrm{th}}\right) \\
& =\operatorname{Pr}\left(\gamma_{0}^{\mathrm{D}}<\gamma_{\mathrm{th}}, \gamma_{1}^{\mathrm{R}}<\gamma_{\mathrm{th}}, \ldots, \gamma_{\mathrm{N}}^{\mathrm{R}}<\gamma_{\mathrm{th}}\right) \\
& =\operatorname{Pr}\left(\gamma_{0}^{\mathrm{D}}<\gamma_{\mathrm{th}}\right) \prod_{j=1}^{N} \operatorname{Pr}\left(\gamma_{j}^{\mathrm{R}}<\gamma_{\mathrm{th}}\right)
\end{aligned}
$$

Substituting (13) and (20) into (22), we can further obtain

$$
\begin{aligned}
& \delta(\rho, \theta)=\left[1-\frac{1}{\sqrt{\pi} \Gamma\left(m_{0}\right)} \sum_{n=1}^{N_{n}} H_{x_{n}} \Gamma\left(m_{0}, \frac{m_{0} \gamma_{h}}{a_{n}(\rho, \theta)}\right)\right]
\end{aligned}
$$

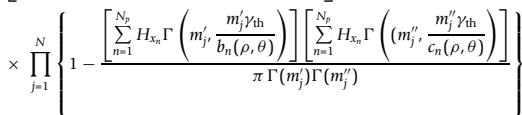


It should be noted that, the outage probability in (23) is a function of the position of MS, i.e., given a specific position of the MS, a corresponding outage probability can be obtained. Theoretically, the distribution of MSs has a strong impact on the system outage probability. Further, assume that $r(\rho, \theta)$ (in polar coordinates) is the PDF, which can be used to describe the distribution of MSs in the cell. Therefore, the system outage probability can be expressed as

$$
\begin{aligned}
P_{\text {out }} & =E_{\rho, \theta}[\delta(\rho, \theta)] \\
& =\int_{0}^{2 \pi} \int_{0}^{R} \delta(\rho, \theta) r(\rho, \theta) \rho \mathrm{d} \rho \mathrm{d} \theta
\end{aligned}
$$

Since the distribution of MSs is arbitrary, the expression in (24) is complex and usually has no closed-form solution. In this section, by making use of the twodimensional composite Simpson's rule [29], the final uplink system outage probability can be approximated as

$$
\begin{aligned}
& P_{\text {out }} \cong \frac{h k}{9} \sum_{p=0}^{P} \sum_{q=0}^{Q}\left[c_{p, q}, \rho_{p} r\left(\rho_{p}, \theta_{q}\right) \delta\left(\rho_{p}, \theta_{q}\right)\right] \\
& \cong \frac{h k}{9} \sum_{p=0}^{p} \sum_{q=0}^{Q}\left\{c_{p, q} \rho_{p} r\left(\rho_{p}, \theta_{q}\right)\left[1-\frac{1}{\sqrt{\pi} \Gamma\left(m_{0}\right)} \sum_{n=1}^{N_{p}} H_{x_{n}} \Gamma\left(m_{0}, \frac{m_{0} \gamma_{t h}}{a_{n}\left(\rho_{p}, \theta_{q}\right)}\right)\right]\right.
\end{aligned}
$$

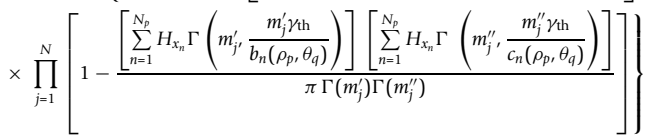

where the two even number $P$ and $Q$ are chosen to determine the step sizes $h=R / P$ and $k=2 \pi / Q$, respectively. In addition, $\rho_{p}=p h,(p=0,1,2, \ldots, P)$ and $\theta_{q}=$ $q k,(q=0,1,2, \ldots, Q)$.

The weigh factor $c_{p, q}$ is element of matrix $\mathbf{C}$, in the $(p$ $+1)^{\text {th }}$ row and $(q+1)^{\text {th }}$ column. Notably, the element in matrix $\mathbf{C}$ can be found in [[29], p. 206].

\section{Numerical results}

In this section, both the Monte Carlo simulation results and theoretical results will be presented. Here, the accuracy of the expression of system outage probability will be verified, and the impacts of path loss exponent, the number of RSs and the distribution of MSs on the system outage probability will be discussed. In addition, the comparison of the outage probability performance between multi-hop cellular system and conventional cellular system will also be shown.

Without loss of generality, an uplink of a single cell multi-hop cellular system is used as a test system. In this system, the BS is in the center of the cell, and the RSs are evenly and symmetrically placed in the cell, that is, the distances between each RS and the BS are the same, and the angles between every two neighboring RSs are also the same. For the sake of simplicity, some parameters of the MS-to-BS link, the MS-to-RS links and the RS-to-BS links are assumed to be the same, i.e., $E=E_{\mathrm{M}}=E_{\mathrm{R}}, m=m_{i}, \beta=\beta_{i}, \sigma=\sigma_{i}$, for $i=0,1, \ldots, 2 N$.

Further, in order to describe the non-uniformity of MSs in the cell, we divide the whole cell into two regions, as shown in Figure 2. Region 1 (denoted as $\Psi_{1}$ ) is the circular area, which is in the center of the cell and with a radius of $R_{h}$. And the residual annular zone is region 2 (denoted as $\Psi_{2}$ ). Therefore, without loss of generality, the PDF for describing the distribution of the MSs in the cell can be supposed as

$$
r(\rho, \theta)=\left\{\begin{array}{cl}
\frac{\lambda}{S_{h}}, & (\rho, \theta) \in \Psi_{1} \\
\frac{1-\lambda}{S-S_{h}}, & (\rho, \theta) \in \Psi_{2}
\end{array}\right.
$$

Where $S_{h}$ is the area of region 1 , while $S$ is the area of the whole cell. $\lambda \in[0,1]$ is the probability that MS distributed in region 1 . It can be observed that, the PDF in (26) varies with the value of $\lambda$. When $\lambda=S_{h} / S$, the MSs are uniformly distributed within the cell; when $\lambda>S_{h} / S$, region 1 is the hot zone, most of the MSs are distributed in this region; when $\lambda<S_{h} / S$, the majority of MSs are located in region 2. Particularly, the MSs are all distributed in region 2 when $\lambda=0$, and when $\lambda=1$, all of MSs are located in region 1 . The main parameters used in simulation are listed in Table 1.

Figs. 3, 4, 5, 6 and 7 show the system outage probability versus the transmit $\operatorname{SNR}\left(E / N_{0}\right)$ in different scenarios. It can be observed that, with the increase of $E / N_{0}$, the system outage probabilities in these figures decrease monotonously. Specifically, Figures 3 and 4 illustrate the

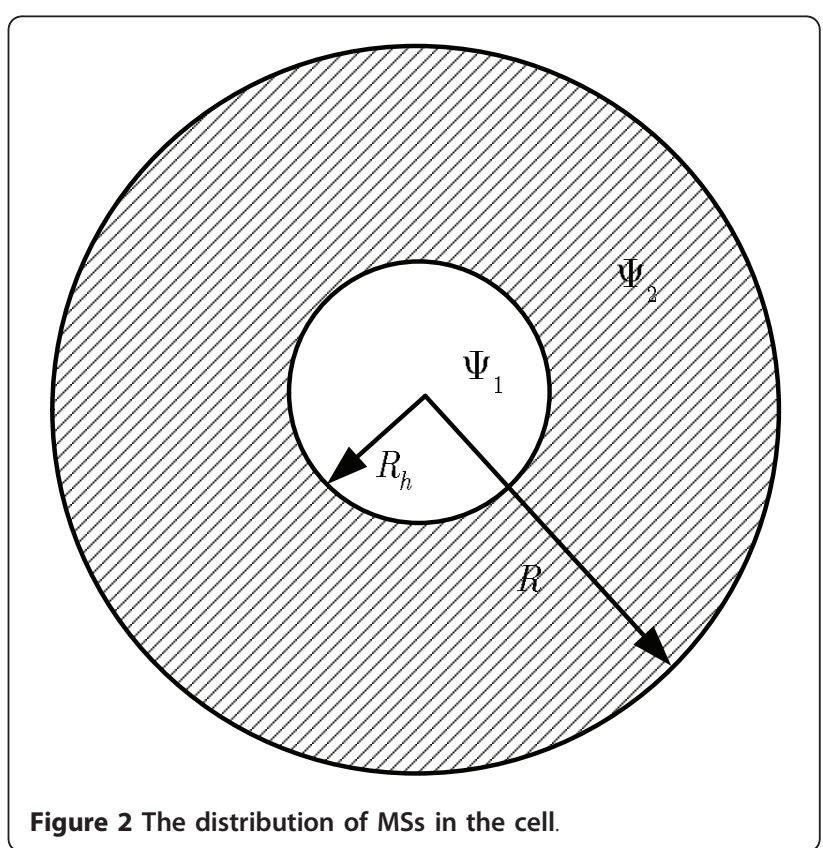


Table 1 Main simulation parameters

\begin{tabular}{lll}
\hline Parameters & Symbol & Value \\
\hline The radius of the cell & $R$ & $400 \mathrm{~m}$ \\
The radius of region 1 & $R_{h}$ & $100 \mathrm{~m}$ \\
The number of RSs & $N$ & 6 \\
The path loss exponent & $\beta$ & 2 \\
The Nakagami factor & $m$ & 1 \\
The standard derivation of lognormal shadowing & $\sigma$ & $12 \mathrm{~dB}$ \\
The reference distance & $d_{0}$ & $50 \mathrm{~m}$ \\
The threshold of SNR & $\gamma_{\text {th }}$ & $0 \mathrm{~dB}$ \\
The number of equidistant nodes for polar radius & $P$ & 8 \\
The number of equidistant nodes for polar angle & $Q$ & 8 \\
The order of Hermite polynomial & $N_{p}$ & 40 \\
The radius of RSs & $A_{i}$ & $267 \mathrm{~m}$ \\
\hline
\end{tabular}

system outage probability as a function of the path loss exponent $\beta$ when the MSs are uniformly distributed $(\lambda$ $\left.=S_{h} / S=0.0625\right)$ and non-uniformly distributed $(\lambda=$ $0.8)$, respectively. These two figures indicate that the system outage performance can be improved with the decrease of $\beta$. That's because the path loss increases with the decrease of $\beta$, and then the channel gain will increase correspondingly. Therefore, the output SNR performance will become better. That is, the number of MSs which cannot satisfy the minimum SNR threshold will be decreased. In other words, the outage performance is enhanced.

Figures 5 and 6 further show the relationship between the system outage probability and the number of RSs $(N)$ when MSs are uniformly distributed $\left(\lambda=S_{h} / S=\right.$ $0.0625)$ and non-uniformly distributed $(\lambda=0.8)$, respectively. Obviously, the value of the system outage probability drops with the increase of $N$. When the value of $N$ is larger, a higher diversity gain can be achieved,

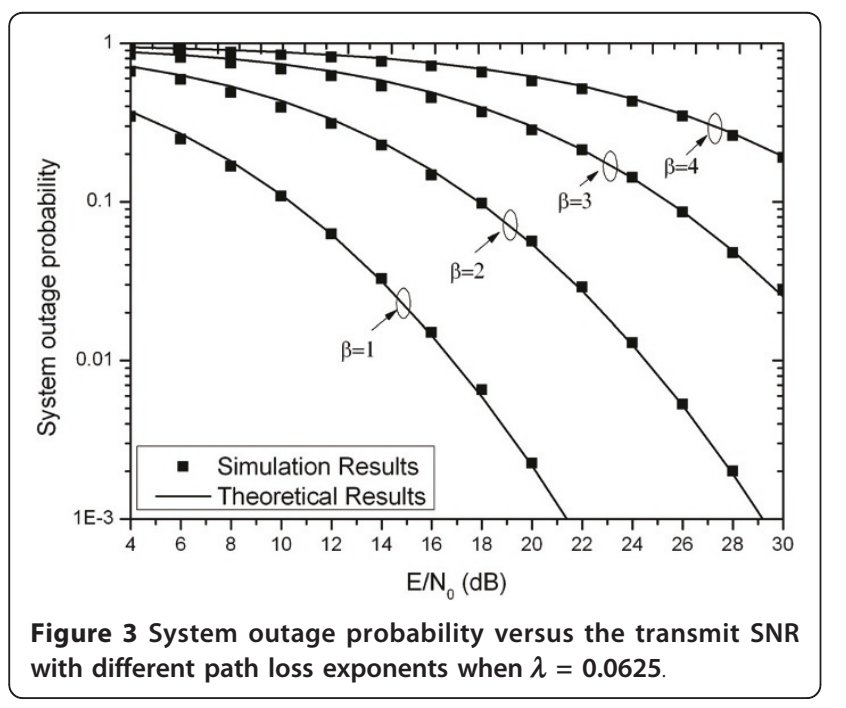

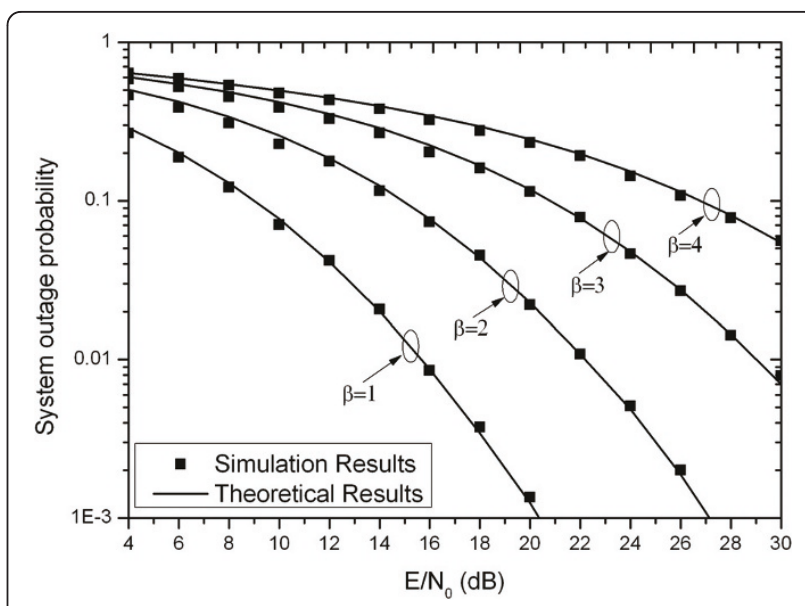

Figure 4 System outage probability versus the transmit SNR with different path loss exponents when $\lambda=0.8$.

which will result in a higher output SNR. Therefore, a better outage performance can be obtained.

Figure 7 illustrates the relationship between system outage probability and the distribution of MSs. It can be observed that, with the increase of $\lambda$, more and more MSs are distributed in region 1 , the average access distance reduces, and this results in the decrease of system outage probability. It also indicates that, the outage probability varies with the value of $\lambda$. Therefore, the distribution of MSs has a strong impact on the system outage probability.

It should also be noted from Figures 3, 4, 5, 6 and 7 that, when the outage probability is below $10^{-1}$, the differences between the theoretical results and simulation results are small enough and can be ignored. Therefore, the expression of the system outage probability shown in this paper can provide perfect approximation to the realistic outage performance of multi-hop cellular system without time-intensive simulations. Further, this expression can be used to evaluate the system outage probability in different scenarios, and it will lay a very good foundation for further research such as RSs placement and network planning.

In conventional cellular system, there is no RS at all in the system and all information bits are transmitted directly between BS and MS. However, information bits in the multi-hop cellular system, in addition to be directly transmitted between BS and MS, can also be indirectly transmitted hop by hop with the help of RSs. Here, we will further compare the system performance between multi-hop cellular system and conventional cellular system. Figure 8 depicts such comparison under different distributions of MSs. Given a specific value of $\lambda$, it can be observed that the outage probability of multi-hop cellular system is smaller than that of conventional cellular system. In other words, the multi-hop 


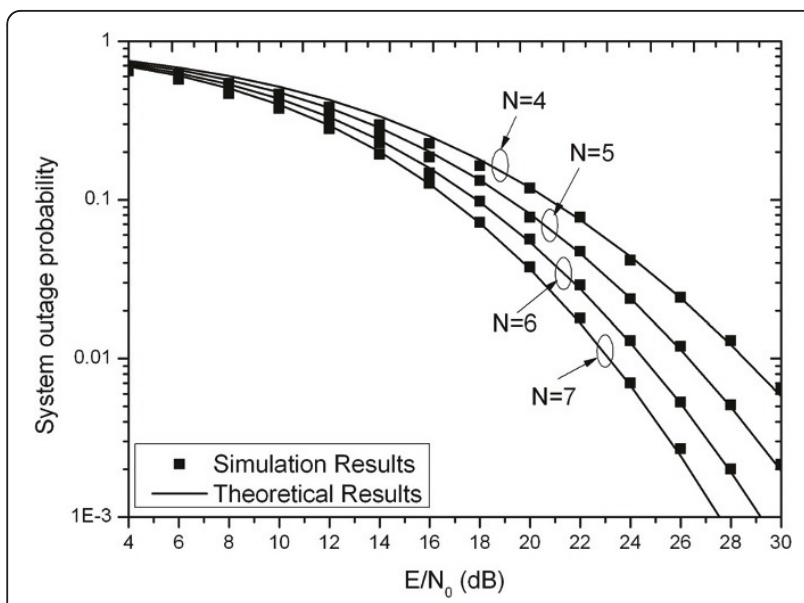

Figure 5 System outage probability versus the transmit SNR with different RSs numbers when $\lambda=0.0625$.

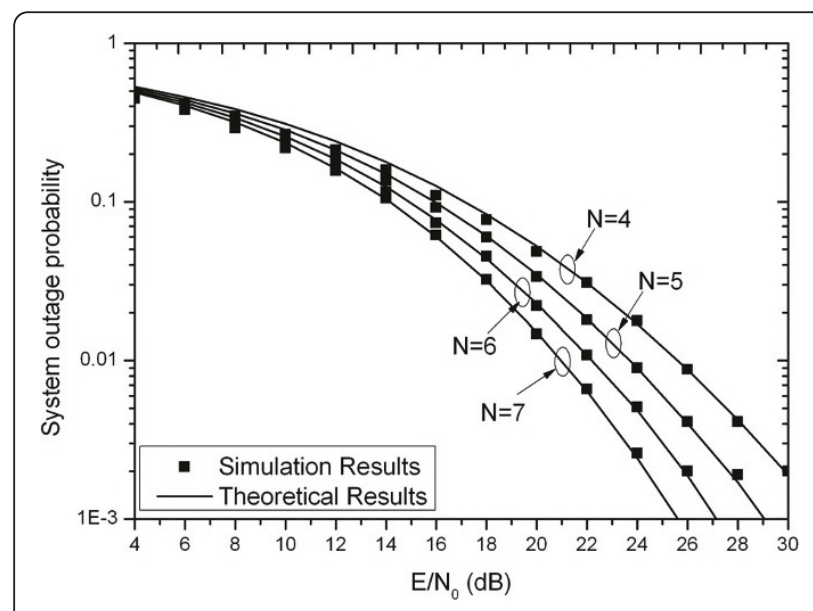

Figure 6 System outage probability versus the transmit SNR with different RSs numbers when $\lambda=0.8$.

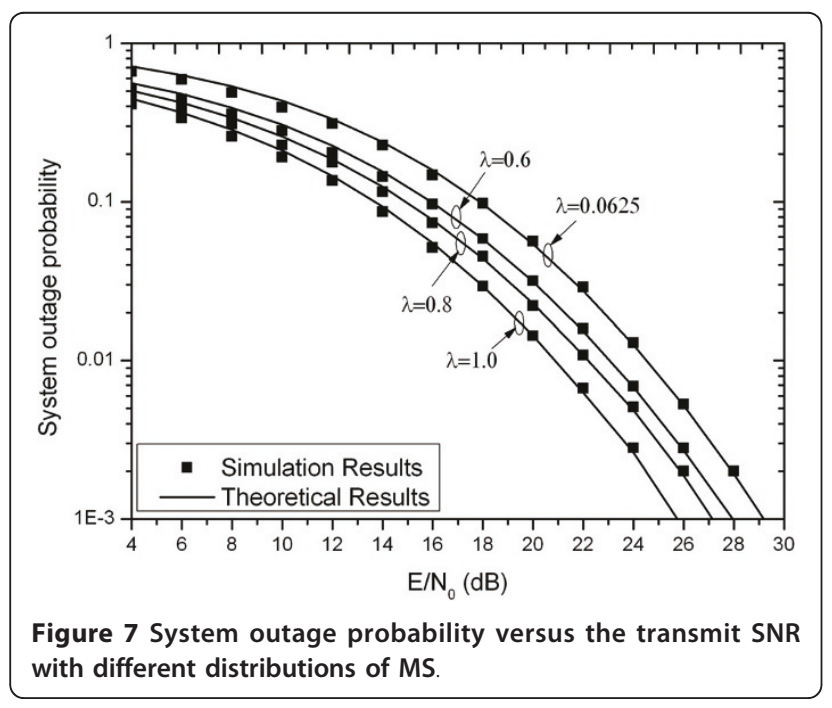

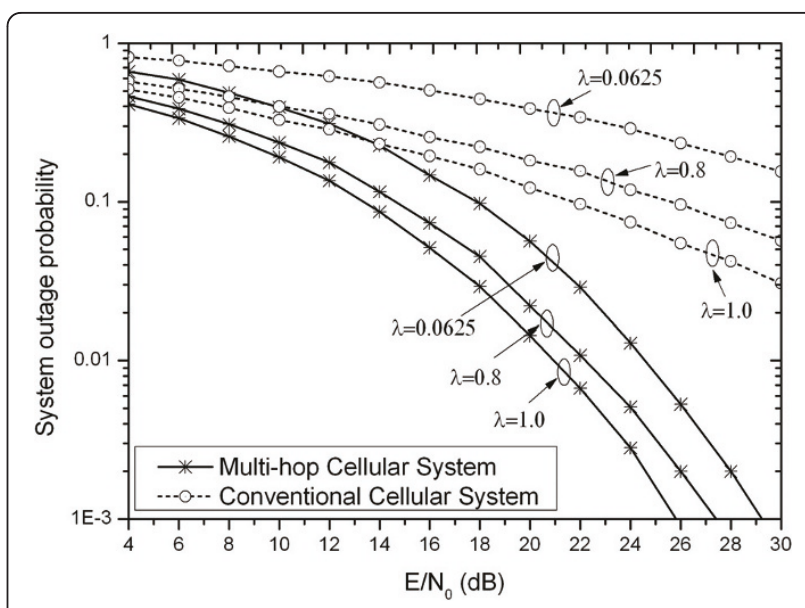

Figure 8 System outage probability performance comparison between multi-hop cellular system and conventional cellular system.

cellular system in this paper always outperforms the conventional cellular system in terms of outage probability.

\section{Conclusion}

In this paper, the system outage probability of the uplink multi-hop cellular system over shad-owed Nakagami- $m$ fading channels is investigated. We firstly introduce the channel model which addresses path loss, lognormal shadowing as well as fast fading. Then, by using Gauss-Hermite integral, we analyze the outage probabilities of direct transmission channel and relay transmission channels, respectively. After that, a theoretical expression of system outage probability is derived after employing the ST scheme and using composite Simpson's rule. Numerical results show that the derived numerical expression is quite accurate to evaluate the outage performance of multi-hop cellular system, and further prove that the multi-hop cellular system in this paper can provide a significant performance gain over the conventional cellular system.

\section{Abbreviations}

AF: amplify-and-forward; BS: base station; CSI: channel state information; MS: mobile station; PDF: probability density function; RSs: relay stations; SNR: signal-to-noise ratio; ST: selective transmission.

\section{Acknowledgements}

This work is supported by the 973 Program of China (2010CB328000), the National Natural Science Foundation of China (61073168 \& 60972023), National Science and Technology Important Special Project (2010ZX03003002 \& 2010ZX03003-004), China Postdoctoral Science Foundation funded project (20110490389), Research Fund of National Mobile Communications Research Laboratory, Southeast University (2010A06), the open research fund of National Mobile Communications Research Laboratory, Southeast University (2010D01), the open research fund of the State Key Laboratory of Integrated Services Networks, Xidian University (ISN12-11), the open research fund of State Key Laboratory of Advanced Optical Communication Systems 
and Networks (2008SH06), NUAA Research Funding (NS2011013) and the startup fund of Nanjing University of Aeronautics and Astronautics.

\section{Author details}

'Key Laboratory for Information System Security of Ministry of Education, School of Software, Tsinghua University, Beijing 100084, China ${ }^{2}$ State Key Laboratory of Integrated Services Networks, Xidian University, Xi'an 710071, China ${ }^{3}$ Computer Science and Engineering Department, Hong Kong University of Science and Technology, Jiulong, Hongkong 999077, China ${ }^{4}$ College of Electronic and Information Engineering, Nanjing University of Aeronautics and Astronautics, Nanjing 210016, China ${ }^{5}$ National Mobile Communications Research Laboratory, Southeast University, Nanjing 210096, China

\section{Competing interests}

The authors declare that they have no competing interests.

Received: 12 January 2011 Accepted: 12 July 2011

Published: 12 July 2011

\section{References}

1. KR Jacobsom, WA Krzymien, System design and thoughput analysis for multihop relaying in cellular systems. IEEE Trans Veh Technol. 58(8), 4514-4528 (2009)

2. A Fujiwara, S Takeda, H Yoshino, T Otsu, Area coverage and capacity enhancement by multihop connection of cdma cellular network, in IEEE 56th Vehicular Technology Conference, vol. 4. (2002), pp. 2371-2374

3. J Cho, ZJ Haas, On the throughput enhancement of the downstream channel in cellular radio networks through multihop relaying. IEEE J Sel Areas Commun. 22(7), 1206-1219 (2004). doi:10.1109/JSAC.2004.829340

4. E Kudoh, F Adachi, Transmit power efficiency of a multi-hop virtual cellular system, in IEEE 58th Vehicular Technology Conference, vol. 5. (2003), pp. 2910-2914

5. J-Y Song, H Lee, D-H Cho, Power consumption reduction by multi-hop transmission in cellular networks, in IEEE 60th Vehicular Technology Conference, vol. 5. (2004), pp. 3120-3124

6. MO Hasna, M-S Alouini, Harmonic mean and end-to-end performance of transmission systems with relays. IEEE Trans Commun. 52(1), 130-135 (2004). doi:10.1109/TCOMM.2003.822185

7. JN Laneman, DNC Tse, GW Wornell, Cooperative diversity in wireless networks: efficient protocols and outage behaviour. IEEE Trans Inf Theory. 50(12), 3062-3080 (2004). doi:10.1109/TIT.2004.838089

8. MO Hasna, M-S Alouini, End-to-end performance of transmission systems with relays over Rayleigh-fading channels. IEEE Trans Wirel Commun. 2(6), 1126-1131 (2003). doi:10.1109/TWC.2003.819030

9. MO Hasna, M-S Alouini, Performance analysis of two-hop relayed transmission over Rayleigh-fading channels, in IEEE Vehicular Technology Conference (VTC02), (Vancouver, BC, Canada, 2002), pp. 1992-1996

10. H Min, S Lee, K Kwak, D Hong, Effect of multiple antennas at the source on outage probability for amplify-and-forward relaying systems. IEEE Trans Wirel Commun. 8(2), 633-637 (2009)

11. JN Laneman, Network coding gain of cooperative diversity, in IEEE MILCOM 2004, (Monterey, CA, 2004), pp. 106-112

12. PA Anghel, M Kaveh, Exact symbol error probability of a cooperative network in a Rayleigh-fading environment. IEEE Trans Wirel Commun. 3(5), 1416-1421 (2004). doi:10.1109/TWC.2004.833431

13. A Bletsas, H Shin, MZ Win, Cooperative communications with outageoptimal opportunistic relaying. IEEE Trans Wirel Commun. 6(9), 3450-3460 (2007)

14. AS Avestimehr, DNC Tse, Outage capacity of the fading relay channel in the low-SNR regime. IEEE Trans Inf Theory. 53(4), 1401-1415 (2007)

15. G Atia, M Sharif, V Saligrama, On optimal outage in relay channels with general fading distributions. IEEE Trans Inf Theory. 53(10), 3786-3797 (2007)

16. A Ribeiro, X Cai, GB Giannikis, Symbol error probabilities for general cooperative links. IEEE Trans Wirel Commun. 4(3), 1264-1273 (2005)

17. $\mathrm{I}-\mathrm{M} \mathrm{Kim}, \mathrm{Z}$ Yi, M Ju, H-K Song, Exact SNR analysis in multi-hop cooperative diversity networks, in IEEE CCECE 2008, Niagara Falls, pp. 843-846 (May 2008)

18. AK Sadek, W Su, KJ Ray Liu, Multinode cooperative communications in wireless networks. IEEE Trans Signal Process. 55(1), 341-351 (2007)
19. C Conne, M Ju, Z Yi, H-K Song, I-M Kim, SER analysis and PDF derivation for multi-hop amplify-and-forward relay systems. IEEE Trans Commun. 58(8), 2413-2424 (2008)

20. Z Yi, M Ju, H-K Song, I-M Kim, Relay ordering in a multi-hop cooperative diversity network. IEEE Trans Commun. 57(9), 2590-2596 (2009)

21. KJR Liu, AK Sadek, W Su, A Kwasinski, Cooperative Communications and Networking (Cambridge University Press, New York, 2009)

22. C Conne, IM Kim, Outage probability of multi-hop amplify-and-forward relay systems. IEEE Trans Wirel Commun. 9(3), 1139-1149 (2010)

23. K Yamamoto, A Kusuda, S Yoshida, Impact of shadowing correlation on coverage of multihop cellular systems, in IEEE International Conference on Communications, vol. 10. (2006), pp. 4538-4542

24. A Goldsmith, Wireless Communication, (Cambridge University Press, New York, 2005)

25. MK Simon, M-S Alouini, Digital Communication over Fading Channels, 2nd edn. (Wiley, New York, 2005)

26. M Abramowitz, IA Stegun, Handbook of Mathematical Functions with Formulas, Graphs, and Mathematical Tables, 9th edn. (Dover Publications, New York, 1970)

27. MO Hasna, MS Alouini, A performance study if dual-hop transmissions with fixed gain relays. IEEE Trans Wirel Commun. 3(6), 1963-1968 (2004). doi:10.1109/TWC.2004.837470

28. S Ikki, MH Ahmed, Performance analysis of cooperative diversity wireless networks over nakagami-m fading channel. IEEE Commun Lett. 11(4), 334-336 (2007)

29. RL Burden, JD Faires, Numerical Analysis, 4th edn. (PWS KENT Publishing Company, Boston, 1989)

doi:10.1186/1687-1499-2011-35

Cite this article as: Zhao et al.: System outage probability analysis in uplink multi-hop cellular systems over composite channels. EURASIP Journal on Wireless Communications and Networking 2011 2011:35.

\section{Submit your manuscript to a SpringerOpen ${ }^{\mathcal{O}}$ journal and benefit from:}

- Convenient online submission

- Rigorous peer review

- Immediate publication on acceptance

- Open access: articles freely available online

- High visibility within the field

- Retaining the copyright to your article

Submit your next manuscript at $\gg$ springeropen.com 